\title{
Fibre intake among the Belgian population by sex-age and sex-education groups and its association with BMI and waist circumference
}

\author{
Yi Lin ${ }^{1}$, Inge Huybrechts ${ }^{1 *}$, Stefanie Vandevijvere ${ }^{2}$, Selin Bolca ${ }^{3}$, Willem De Keyzer ${ }^{1,4}$, \\ Stephanie De Vriese ${ }^{5}$, Anja Polet ${ }^{1}$, Melissa De Neve ${ }^{1}$, Herman Van Oyen ${ }^{2}$, John Van Camp ${ }^{6}$, \\ Guy De Backer ${ }^{1}$ and Stefaan De Henauw ${ }^{1,4}$ \\ ${ }^{1}$ Unit Nutrition and Food Safety, Department of Public Health, Faculty of Medicine and Health Sciences, Ghent University, \\ De Pintelaan 185, B-9000 Ghent, Belgium \\ ${ }^{2}$ Unit of Epidemiology, Scientific Institute of Public Health, J. Wytsmanstraat 14, B-1050 Brussels, Belgium \\ ${ }^{3}$ Laboratory for Bioinformatics and Computational Genomics (BIOBIX), Faculty of Bioscience Engineering, Ghent \\ University, Coupure Links 653, B-9000 Ghent, Belgium \\ ${ }^{4}$ Department of Nutrition and Dietetics, Faculty of Health Care Vesalius, University College Ghent, Keramiekstraat 80, \\ B-9000 Ghent, Belgium \\ ${ }^{5}$ Alpro Foundation vzw, Vlamingstraat 28, 8560 Wevelgem, Belgium \\ ${ }^{6}$ Department of Food Safety and Food Quality, Ghent University, Coupure Links 653, B-9000 Ghent, Belgium
}

(Received 4 June 2010 - Revised 5 November 2010 - Accepted 8 November 2010 - First published online 29 March 2011)

\begin{abstract}
The objectives of the present study were to assess total dietary fibre intake and the main contributors to fibre intake in the Belgian population by sex-age and sex-education groups and to investigate its relationship with BMI and waist circumference (WC). The participants of the Belgian food consumption survey (2004) were randomly selected. Information about food intake was collected using two repeated, non-consecutive $24 \mathrm{~h}$ recall interviews. A total of 3083 individuals ( $\geq 15$ years; 1546 men and 1537 women) completed both interviews. The main contributors to total fibre intake $(17.8 \mathrm{~g} / \mathrm{d})$ were cereals and cereal products $(34 \% ; 5.9 \mathrm{~g} / \mathrm{d})$, potatoes and other tubers $(18.6 \% ; 3.3 \mathrm{~g} / \mathrm{d})$, fruits $(14.7 \% ; 2.8 \mathrm{~g} / \mathrm{d})$ and vegetables $(14.4 \% ; 2.6 \mathrm{~g} / \mathrm{d})$. Legume fibre intake was extremely low $(0 \cdot 672 \% ; 0 \cdot 139 \mathrm{~g} / \mathrm{d})$. In all sex-age and sex-education groups, total fibre intake was below the recommendations of the Belgian Superior Health Council. Men $(21 \mathrm{~g} / \mathrm{d})$ consumed significantly more fibre than women $(17 \cdot 3 \mathrm{~g} / \mathrm{d})(P<0 \cdot 001)$. Lower educated men and higher educated women reported the highest fibre intake. A significant inverse association was found between total fibre intake and WC $(\beta=-0 \cdot 118$, $P<0 \cdot 001)$. Fruit-derived fibre was positively associated with WC $(\beta=0 \cdot 731, P=0 \cdot 001)$. In summary, total fibre intake was inversely associated with WC, whereas fruit-derived fibre intake was positively associated with WC in the Belgian population.
\end{abstract}

Key words: Dietary fibre: Food consumption surveys: Waist circumference: BMI

Dietary fibre is one of the nutritional compounds of vegetable foods, defined by the Codex Committee on Nutrition and Foods for Special Dietary Uses as carbohydrate polymers with ten or more monomeric units, which are not hydrolysed by endogenous enzymes in the small intestine ${ }^{(1)}$. Dietary fibre can be classified into water-soluble and water-insoluble fibre. Water-soluble fibres can delay small-bowel absorption, which may reduce cholesterol absorption, but also pancreatic enzyme activity and protein digestion ${ }^{(2,3)}$. Subsequently, the colonic fermentation of fibres results in the production of gases and SCFA ${ }^{(4)}$, which causes a longer-lasting satiety and lowers the glycaemic index of foods, and, consequently, attenuates the insulin response ${ }^{(5,6)}$. Due to its anti-nutritive properties and non-digestibility, water-insoluble fibre can increase the bulkiness of stool and faecal mass, thereby shortening transit time ${ }^{(3)}$.

A decreased dietary fibre intake in Western countries is found to be associated with a higher prevalence of chronic diseases $^{(7,8)}$. Epidemiological data suggest an inverse association between the consumption of dietary fibre and chronic diseases $^{(9)}$ and a positive association with an overall healthier profile (lower blood pressure, lower cholesterol levels and improved insulin sensitivity $)^{(10)}$. In the past decades, the decline in dietary fibre has been hypothesised as a possible determinant for increased adiposity, metabolic disorders and $\mathrm{CVD}^{(11)}$. Several recent studies have reported that dietary fibre intake

Abbreviations: BSHC, Belgian Superior Health Council; WC, waist circumference.

* Corresponding author: I. Huybrechts, fax +32 9332 4994, email inge.huybrechts@ugent.be 
may protect against adiposity and metabolic disorders ${ }^{(12-14)}$. Moreover, recent cohort studies and randomised control trials have shown a significant inverse association between fibre consumption and the risk of developing obesity ${ }^{(15)}$ and the metabolic syndrome ${ }^{(16)}$. Therefore, the intake of dietary fibres is strongly recommended by the WHO $(>25 \mathrm{~g} / \mathrm{d})$ and the Belgian Superior Health Council (BSHC) ( $40 \mathrm{~g} / \mathrm{d}$ for male adolescents (14-18 years); $30 \mathrm{~g} / \mathrm{d}$ for adults and female adolescents $(14-18$ years $))^{(17,18)}$ due to the health benefits.

These fibre recommendations have been translated into food-based dietary guidelines ${ }^{(19)}$ that have been developed to help the general population in choosing a healthy diet. Vandevijvere et $a l .{ }^{(20)}$ compared the Belgian food-based dietary guidelines, which are based on our Belgian dietary recommendations, with the results derived from the Belgian food consumption survey. This comparison showed that Belgian dietary habits deviate importantly from the Belgian dietary guidelines. Mainly, the intake of plant products (fruits and vegetables), which are important for our fibre intake, was very low in Belgium ${ }^{(20)}$. Vandevijvere et al. reported that only 38 and $47 \%$ of the Belgian population consumed vegetables and fruits, respectively, whereas 82 and $52 \%$ of the Belgian population consumed bread and cereals, and potatoes and other tubers, respectively, on a daily basis. Men consumed more bread and cereal products, potatoes and grain products, and fruits than women did, though no differences were found in vegetable intake. The consumption of bread and cereals, and potatoes and grains decreased by increasing age category. The daily consumption of vegetables and fruits, however, was highest among the subpopulation aged 60-74 years old and lowest in the adolescents aged 15-18 years. Furthermore, Huybrechts \& De Henauw ${ }^{(21)}$ showed that the majority of Flemish preschoolers aged $2.5-6.5$ years old did not meet the recommended fibre intake of the BSHC. Matthys et al. ${ }^{(22)}$ reported that Flemish adolescents (13-18 years) also did not reach the fibre requirements of the BSHC.

When considering the above-mentioned gaps in the consumption of plant-rich foods in our Belgian diet and knowing that the prevalence of obesity increased in the past years ${ }^{(23,24)}$, it would be interesting to get more insight into the intake of dietary fibres among our Belgian population and its association with overweight and obesity.

Therefore, the main objectives of the present study were to evaluate dietary fibre intake among the Belgian population and to explore possible differences in intake among sexage and sex-education groups. Furthermore, in view of the health effects of fibres related to obesity, the association between fibre intake and BMI and waist circumference (WC) was assessed.

\section{Materials and methods}

\section{Sampling design and data collection}

For the purpose of the present study, data from the Belgian national food consumption survey were used ${ }^{(25)}$. This survey was performed in 2004 and followed, to a large extent, the recommendations of the European Food Consumption
Survey Method project, defining a method to monitor food consumption in nationally representative samples of all agesex categories ${ }^{(26)}$. The target population was defined as all persons of 15 years and older residing in Belgium and willing to participate. Institutionalised individuals, persons not able to speak one of the national languages or physically or mentally unable to be interviewed were excluded from the survey. A stratified multi-stage sampling design was used to select the participants from the whole country covering regions of Flanders, Brussels and Walloon. The population was stratified in four sex-age groups (15-18, 19-59, 60-74 and $\geq 75$ years). Approximately 400 individuals were included in each sex-age stratum. Adolescents were defined as participants being 15-18 years of age.

The present study was conducted according to the guidelines laid down in the Declaration of Helsinki, and all procedures involving human subjects were approved by the medical ethical committee of the Scientific Institute of Public Health in Brussels. Written or verbal informed consent was obtained from all subjects. Verbal consent was witnessed and formally recorded.

\section{Dietary intake assessment}

Two repeated, non-consecutive $24 \mathrm{~h}$ dietary recall interviews were used to collect the food consumption data. During the first home visit, a $24 \mathrm{~h}$ dietary recall was performed through a computer-assisted face-to-face interview by a well-trained dietitian. The second $24 \mathrm{~h}$ dietary recall was repeated $2-8$ weeks later (median 3 weeks). Interviews were randomly allocated to different days of the week and over a 12-month period in order to include day-to-day and seasonal variations. During the interviews, information (quantities, brand names and recipes used) was collected on all foods and beverages consumed during the preceding day.

The validated software package EPIC-SOFT (International Agency for Research on Cancer (IARC), Lyon, France), designed to obtain a very detailed description and quantification of all foods consumed, was used to obtain standardised $24 \mathrm{~h}$ dietary recall interviews ${ }^{(27)}$. Additionally, a book with coloured photographs of foods in different portion sizes was used to support the quantification.

In the present study, 2288 different food items (from seventeen food groups and 107 food subgroups) were consumed by the respondents. Dietary fibre intake was estimated based on the American Association of Analytical Chemists method ${ }^{(28)}$ used in the Belgian NUBEL ${ }^{(29)}$, the Dutch $\mathrm{NEVO}^{(30)}$ and the USDA food composition databases ${ }^{(31)}$. Dietary fibre, as defined by the American Association of Analytical Chemists, includes NSP and indigestible carbohydrates resistant to digestive enzymes. Energy-adjusted dietary fibre intake was also calculated using the formula total fibre intake $(\mathrm{g} / \mathrm{d}) /$ total energy intake $(\mathrm{MJ} / \mathrm{d})$.

\section{Level of education}

Participants were asked to report the highest degree that they had obtained during the first interview. Four categories of 
education level were created: (1) lower secondary; (2) vocational, technical or art; (3) general secondary; (4) higher education (bachelor, master or above).

\section{Anthropometric measurements}

Weight $(\mathrm{kg})$ and height $(\mathrm{m})$ were self-reported by the respondents, while WC was measured by the trained dietitians at home after $24 \mathrm{~h}$ recall interviews. Pregnant women reported their weight before pregnancy. BMI was calculated as weight $(\mathrm{kg}) /$ height $\left(\mathrm{m}^{2}\right)$. Adult participants were classified into four BMI categories according to the WHO definition: underweight $\left(<18.5 \mathrm{~kg} / \mathrm{m}^{2}\right)$; normal weight $\left(18.5-24.9 \mathrm{~kg} / \mathrm{m}^{2}\right)$; overweight $\left(25 \cdot 0-29 \cdot 9 \mathrm{~kg} / \mathrm{m}^{2}\right)$; obesity $\left(\geq 30 \cdot 0 \mathrm{~kg} / \mathrm{m}^{2}\right)^{(32)}$. The BMI of adolescent participants was classified into the same four categories according to Cole et al. ${ }^{(33)}$ (normal weight, overweight and obesity) and Flemish cut-off values (for underweight only) ${ }^{(34)}$. The cut-off criteria for adults' WC were as follows: normal, $<80 \mathrm{~cm}$ (women) and $<94 \mathrm{~cm}$ (men); normal to borderline, $80-88 \mathrm{~cm}$ (women) and $94-102 \mathrm{~cm}$ (men); abdominal obesity, $\geq 88 \mathrm{~cm}$ (women) and $\geq 102 \mathrm{~cm}$ (men) $)^{(35)}$. The cut-off criteria of WC for adolescents were based on Taylor et al. ${ }^{(36)}$.

\section{Statistical analysis}

Descriptive and statistical analyses by the sex-age and sexeducation groups were performed using SPSS for Windows version 15 (SPSS, Inc., Chicago, IL, USA). Results were considered statistically significant at an $\alpha$ two-tailed level of $0 \cdot 05$. Tests for normality and equality of the variances were performed using the Kolmogorov-Smirnov and Levene's test, respectively. To obtain a normal distribution, the dietary total fibre intakes and total fibre intake from main sources were log-transformed. Descriptive statistics include mean intakes of total fibre and food group-specific fibre intakes, BMI and WC with their standard errors. Student's $t$ test, ANOVA with Bonferroni correction was used to compare means between the groups.

Associations between BMI or WC (separate dependent variables) and total fibre intake or food group-specific fibre intake (independent variables) were investigated by stepwise multiple linear regression via three models: (1) unadjusted model; (2) model adjusted for age, sex, region and education level; (3) model further adjusted for total energy intake and the interactions based on model 2. The null hypothesis posited that there is no association between fibre intake and the BMI or WC $\left(H_{0}: \beta=0\right)$. The potential effect of confounding factors such as age, sex, region and education level was analysed by stratification to obtain the second coefficient estimate ( $\beta$ in model 2 ). Additionally, interactions between the independent variable and all effect factors and between the effect factors were examined, resulting in the third coefficient estimate ( $\beta$ from model 3 ). Total fibre and food group-specific fibre intakes were investigated in separate models because of collinearity. Outliers were removed according to the residual method.

\section{Results}

\section{Study population}

In total, 3083 individuals (1546 men and 1537 women) out of 7543 contacted individuals participated in the survey and completed both $24 \mathrm{~h}$ dietary recalls. Among all 3083 individuals, 2961 participants reported their education level, 3055 reported their weight and height, and for 2875 subjects, WC was measured.

BMI and WC of all participants differed according to sex, age and education level (Table 1). The prevalence of overweight or obese men was higher compared with women, with a mean BMI of 25 and $24 \mathrm{~kg} / \mathrm{m}^{2}$, respectively. Of all the participants, $42 \%$ of women and $29 \%$ of men were abdominally obese. The prevalence of overweight or obese subjects, based on both BMI and WC, was the highest in the groups of $60-75$ and $\geq 75$ years and in the subjects with the lowest educational level.

\section{Total fibre intake}

The mean total fibre intake in Belgium was $17 \cdot 8(1 \cdot 4-57) \mathrm{g} / \mathrm{d}$. Men consumed significantly more fibre than women $(P<0.001)$, with the lowest and highest intakes reported by the 15-18 and the 60-74 years of sex-age groups, respectively (Table 2). With regard to the level of education, men in the lowest education group and women with a higher education level had the highest mean total fibre intakes (21 and $16.7 \mathrm{~g} / \mathrm{d}$, respectively). The energy-adjusted dietary fibre intakes, on the other hand, were significantly higher in women compared with men $(P<0 \cdot 001)$, increased by ageing and was the highest in the lowest educated women in the sex-education groups.

The mean dietary fibre intake of the Belgian population was approximately half of the recommended intake by the BSHC (Table 2$)^{(17)}$, with the majority (63\% men and $89 \%$ women) not meeting the recommendations. Fibre intakes of Belgian adults were too low according to several international guidelines for adults, such as the WHO $(>25 \mathrm{~g} / \mathrm{d})^{(18)}$, the USDA (men, $38 \mathrm{~g} / \mathrm{d}$; women, $25 \mathrm{~g} / \mathrm{d})^{(31)}$, the Institute of Medicine of the National Academies (men aged $19-50$ years, $38 \mathrm{~g} / \mathrm{d}$; men $\geq 50$ years, $30 \mathrm{~g} / \mathrm{d}$; women aged $19-50$ years, $25 \mathrm{~g} / \mathrm{d}$; women $\geq 50$ years, $21 \mathrm{~g} / \mathrm{d})^{(37)}$ and the British Nutrition Foundation $(18.0 \mathrm{~g} / \mathrm{d}$ NSP, approximately $24 \mathrm{~g} / \mathrm{d}$ total dietary fibre) ${ }^{(38)}$. Likewise, the adolescent population (men, $17 \cdot 8 \mathrm{~g} / \mathrm{d}$; women, $15.0 \mathrm{~g} / \mathrm{d}$ ) reported intakes below the dietary reference intakes of the Institute of Medicine of the National Academies (men, 38g/d; women, $26 \mathrm{~g} / \mathrm{d})^{(37)}$ and Williams' Age plus 5 guideline $(19 \cdot 0-23 \mathrm{~g} / \mathrm{d})^{(39)}$.

\section{Main food groups contributing to fibre intake}

Five food groups contributed to $82 \%$ of the total fibre intake in Belgium (corresponding with a mean intake of $14.7 \mathrm{~g} / \mathrm{d}$ ). In all sex-age (Table 3) and sex-education (Table 4) groups, cereals and cereal products contributed most (34\%; $5.9 \mathrm{~g} / \mathrm{d}$ ), with bread, crispbread and rusks as the main sources within this category, followed by potatoes and other tubers 
Table 1. Characteristics of the participants of the Belgian national food consumption survey (2004-5)

(Mean values with their standard errors)

\begin{tabular}{|c|c|c|c|c|c|c|c|c|c|c|c|c|}
\hline \multirow[b]{3}{*}{ Characteristics } & \multirow{3}{*}{$\begin{array}{c}\text { Population } \\
n\end{array}$} & \multicolumn{6}{|c|}{$\operatorname{BMI}\left(\mathrm{kg} / \mathrm{m}^{2}\right)^{*}$} & \multicolumn{5}{|c|}{ Waist circumference $(\mathrm{cm}) \dagger$} \\
\hline & & \multirow[b]{2}{*}{ Mean } & \multirow[b]{2}{*}{ SEM } & \multicolumn{4}{|c|}{ Prevalence (\%) } & \multirow[b]{2}{*}{ Mean } & \multirow[b]{2}{*}{ SEM } & \multicolumn{3}{|c|}{ Prevalence (\%) } \\
\hline & & & & Underweight & Normal & Overweight & Obesity & & & Normal & Borderline & Abdominal obesity \\
\hline Sex & 3083 & & & & & & & & & & & \\
\hline Men & 1546 & 25 & 0.1 & $3 \cdot 1$ & 52 & 34 & $10 \cdot 1$ & 88 & 0.7 & 43 & 27 & 29 \\
\hline Women & 1537 & 24 & 0.1 & $5 \cdot 7$ & 58 & 25 & $10 \cdot 5$ & 80 & 0.7 & 39 & 19 & 42 \\
\hline Age (years) & 3083 & & & & & & & & & & & \\
\hline $15-18$ & 762 & 21 & 0.1 & $9 \cdot 7$ & 79 & $10 \cdot 2$ & $1 \cdot 3$ & 76 & 0.6 & 72 & 20 & $7 \cdot 6$ \\
\hline $19-59$ & 828 & 24 & 0.2 & 3.7 & 60 & 26 & $10 \cdot 1$ & 81 & 1.0 & 51 & 24 & 25 \\
\hline $60-75$ & 789 & 26 & 0.2 & $1 \cdot 0$ & 36 & 44 & $18 \cdot 6$ & 91 & 0.9 & 20 & 27 & 53 \\
\hline$\geq 75$ & 704 & 25 & 0.2 & $3 \cdot 2$ & 46 & 40 & $10 \cdot 8$ & 90 & 1.2 & 19 & 22 & 59 \\
\hline Education & 2961 & & & & & & & & & & & \\
\hline Lower secondary or less & 967 & 26 & 0.1 & $2 \cdot 2$ & 41 & 40 & $16 \cdot 5$ & 97 & 0.4 & 21 & 23 & 56 \\
\hline Vocational, technical or art & 734 & 24 & 0.2 & 5.4 & 60 & 27 & $7 \cdot 6$ & 87 & 0.5 & 49 & 26 & 25 \\
\hline General secondary & 530 & 22 & 0.2 & $6 \cdot 0$ & 70 & 20 & 4.2 & 83 & 0.6 & 57 & 22 & 21 \\
\hline Higher & 730 & 24 & 0.2 & 4.7 & 60 & 27 & 8.6 & 89 & 0.5 & 47 & 23 & 30 \\
\hline \multicolumn{13}{|l|}{ Region } \\
\hline Flanders & 955 & 24 & 0.1 & 3.7 & 58 & 29 & 9.5 & 84 & 0.6 & 41 & 24 & 35 \\
\hline Brussels & 233 & 24 & 0.3 & 6.5 & 58 & 27 & $7 \cdot 8$ & 83 & 1.5 & 43 & 28 & 29 \\
\hline Walloon & 918 & 25 & 0.1 & $5 \cdot 1$ & 50 & 33 & $12 \cdot 5$ & 86 & 0.8 & 40 & 21 & 38 \\
\hline
\end{tabular}

${ }^{*}$ BMI categories of adults: underweight, $<18.5 \mathrm{~kg} / \mathrm{m}^{2} ;$ normal weight, $18.5-24.9 \mathrm{~kg} / \mathrm{m}^{2} ;$ overweight, $25.0-29.9 \mathrm{~kg} / \mathrm{m}^{2} ;$ obesity, $\geq 30.0 \mathrm{~kg} / \mathrm{m}^{2}(32)$. For adolescents, the categories were assigned according to the cut-off values for children and adolescents developed by Cole et al. ${ }^{(33)}$ and according to the Flanders growth charts ${ }^{(34)}$.

† Waist circumference categories for adults: normal, $<94 \mathrm{~cm}$ (men) and $<80 \mathrm{~cm}$ (women); borderline, $94-102 \mathrm{~cm}$ (men) and $80-88 \mathrm{~cm}$ (women); obesity, $\geq 102 \mathrm{~cm}$ (men) and $\geq 88 \mathrm{~cm}$ (women) ${ }^{(35)}$. For adolescents, the categories were assigned according to the cut-off values given by Taylor et al. ${ }^{(36)}$ 
Table 2. Reported and recommended daily total dietary fibre intakes and the percentage of the subjects in the agreement with the recommendations of the Belgian Superior Health Council(17), stratified in sex-age and sex-education groups

(Mean values with their standard errors)

\begin{tabular}{|c|c|c|c|c|c|c|c|c|c|c|c|c|}
\hline \multirow[b]{4}{*}{ Stratification } & \multicolumn{10}{|c|}{ Total fibre intake } & & \\
\hline & \multicolumn{5}{|c|}{ Men } & \multicolumn{5}{|c|}{ Women ${ }^{*}$} & \multicolumn{2}{|c|}{ Subjects (\%)† } \\
\hline & \multicolumn{2}{|c|}{ Reported (g/d) } & \multicolumn{2}{|c|}{$\begin{array}{l}\text { Energy-adjusted } \\
\qquad(\mathrm{g} /(\mathrm{MJ} \times \mathrm{d}))\end{array}$} & \multirow[b]{2}{*}{ Recommended (g/d) } & \multicolumn{2}{|c|}{ Reported (g/d) } & \multicolumn{2}{|c|}{$\begin{array}{l}\text { Energy-adjusted } \\
\qquad(\mathrm{g} /(\mathrm{MJ} \times \mathrm{d}))\end{array}$} & \multirow[b]{2}{*}{ Recommended (g/d) } & \multirow[b]{2}{*}{ Men } & \multirow[b]{2}{*}{ Women } \\
\hline & Mean & SEM & Mean & SEM & & Mean & SEM & Mean & SEM & & & \\
\hline \multicolumn{13}{|l|}{ Age (years) } \\
\hline $15-18$ & $17 \cdot 8^{\mathrm{a}}$ & 0.4 & $1.7^{\mathrm{a}}$ & 0.1 & 40 & $15 \cdot 0^{\mathrm{a}}$ & 0.3 & $2 \cdot 1^{\mathrm{a}}$ & 0.1 & 30 & 0.0 & 0.8 \\
\hline $19-59$ & $19 \cdot 5^{\mathrm{a}}$ & 0.4 & $2 \cdot 0^{\mathrm{b}}$ & 0.1 & 30 & $15 \cdot 8^{\mathrm{a}}$ & 0.3 & $2 \cdot 4^{b}$ & 0.1 & 30 & $12 \cdot 4$ & $2 \cdot 1$ \\
\hline $60-75$ & $21^{\mathrm{b}}$ & 0.4 & $2 \cdot 4^{\mathrm{c}}$ & 0.1 & 30 & $17 \cdot 5^{\mathrm{b}}$ & 0.4 & $2 \cdot 8^{\mathrm{c}}$ & 0.1 & 30 & $14 \cdot 3$ & $5 \cdot 6$ \\
\hline$\geq 75$ & $19 \cdot 8^{\mathrm{a}}$ & 0.4 & $2 \cdot 5^{\mathrm{c}}$ & 0.1 & 30 & $16 \cdot 2^{\mathrm{a}}$ & 0.4 & $2 \cdot 7^{\mathrm{c}}$ & 0.1 & 30 & $10 \cdot 2$ & $2 \cdot 7$ \\
\hline \multicolumn{13}{|l|}{ Education } \\
\hline Lower secondary or less & $21^{a}$ & 0.4 & $2 \cdot 5^{\mathrm{a}}$ & 0.1 & 30 & $16 \cdot 3^{a}$ & 0.3 & $2 \cdot 6^{a}$ & 0.1 & 30 & 14.6 & 3.5 \\
\hline $\begin{array}{l}\text { Vocational, technical or } \\
\text { art education }\end{array}$ & $19 \cdot 1^{\mathrm{b}}$ & 0.4 & $2 \cdot 0^{\mathrm{b}}$ & 0.1 & 30 & $15 \cdot 1^{a}$ & 0.3 & $2 \cdot 3^{b}$ & 0.1 & 30 & 9.6 & 0.9 \\
\hline $\begin{array}{l}\text { General secondary } \\
\text { education }\end{array}$ & $18 \cdot 8^{a}$ & 0.5 & $2 \cdot 0^{\mathrm{b}}$ & 0.1 & 30 & $16 \cdot 5^{\mathrm{b}}$ & 0.4 & $2 \cdot 3^{b}$ & 0.1 & 30 & $6 \cdot 3$ & $3 \cdot 2$ \\
\hline Higher education & $19 \cdot 1^{\mathrm{a}}$ & 0.4 & $2 \cdot 1^{\mathrm{b}}$ & 0.1 & 30 & $16 \cdot 7^{\mathrm{b}}$ & 0.3 & $2 \cdot 5^{\mathrm{a}}$ & 0.1 & 30 & 1.1 & 3 \\
\hline
\end{tabular}

${ }^{a, b, c} \mathrm{c}$ Mean values within a column with unlike superscript letters were significantly different $(P<0.05$, ANOVA with Bonferroni correction after log transformation).

* Mean values were significantly different between men and women $(P<0.05$; Student's $t$ test after log transformation).

†Percentage of participants meeting the recommended dietary fibre intake of the Belgian Health Superior Council. 
$(18 \cdot 6 \%, 3 \cdot 3 \mathrm{~g} / \mathrm{d})$, fruits $(14 \cdot 7 \%, 2 \cdot 8 \mathrm{~g} / \mathrm{d})$ and vegetables $(14.4 \%$, $2.6 \mathrm{~g} / \mathrm{d}$ ). Interestingly, the subgroup 'bread, crispbread and rusks' of cereals and cereal products contributed significantly less in the adolescent group compared with the older groups $(P<0.05)$, whereas 'other cereal products', in contrast, contributed most to adolescents' intake. The intake of legumederived fibres was extremely low $(0.672 \% ; 0.139 \mathrm{~g} / \mathrm{d})$ in all sex-age and sex-education groups. The contributions of fruits and vegetables were the largest in the elderly population (60-74 years in particular) and the lowest in adolescents. In general, potato-derived fibre intakes decreased with the level of education. The contribution of other cereal products was the lowest for lower secondary educated participants, whereas higher educated subjects consumed more fibres from vegetables and fruits.

\section{Anthropometric indices and total and specific fibre intake}

Associations between BMI or WC, on the one hand, and total or food group-specific fibre intake, on the other hand, were investigated by stepwise multiple linear regression (Table 5). In model 1, crude BMI was associated with fibre intake from potatoes and other tubers, vegetables, fruits, and cereals and cereal products. After adjustment for age, sex, region and education level (model 2), a significant association was observed between BMI and the intake of fibres from cereals and cereal products $(\beta=-0.045, P=0.025)$, but no association was found after adjustment for energy intakes and interactions with and between the effect factors (model 3). According to model 3, WC, on the other hand, was inversely related to the total fibre intakes $(\beta=-0 \cdot 118, P<0 \cdot 001)$ and positively related to fruit-derived fibre intakes $(\beta=0.731, P=0.001)$.

\section{Discussion}

\section{Total and food group-specific fibre intake}

The total daily fibre intakes in this large population-based national nutrition survey were on average $17 \cdot 8 \mathrm{~g}$ and below (men 63\%, women 89\%) the recommendations proposed by the $\mathrm{BSHC}^{(17)}$.

Compared with the study reported by Matthys et al. ${ }^{(22)}$ involving Flemish adolescents (13-17 years) living in Ghent and using $7 \mathrm{~d}$ food records, the male adolescents participating in the present study consumed considerably less dietary fibre than Ghent adolescents (mean $21 \mathrm{~g} / \mathrm{d}$ ), whereas the female adolescents from Ghent had a slightly higher average dietary fibre intake (mean $15.9 \mathrm{~g} / \mathrm{d}$ ). After adjustment for energy intake, however, male adolescents from the Belgian food consumption survey had slightly lower intakes than reported in the study of Matthys et al. $(1.8 \mathrm{~g} / \mathrm{MJ} \times \mathrm{d})$, while female adolescents had higher intakes than those in the study of Matthys et al. $(1.9 \mathrm{~g} / 10 \mathrm{MJ})$.

Table 3. Contribution $(\mathrm{g} / \mathrm{d})$ of different food groups to the total fibre intake among the study population, stratified by the sex-age groups (Mean values with their standard errors)

\begin{tabular}{|c|c|c|c|c|c|c|c|c|}
\hline \multirow[b]{3}{*}{ Food sources } & \multicolumn{8}{|c|}{ Age (years) } \\
\hline & \multicolumn{2}{|c|}{$15-18$} & \multicolumn{2}{|c|}{$19-59$} & \multicolumn{2}{|c|}{$60-75$} & \multicolumn{2}{|c|}{$\geq 75$} \\
\hline & Mean & SEM & Mean & SEM & Mean & SEM & Mean & SEM \\
\hline \multicolumn{9}{|l|}{ Men } \\
\hline Total intake of main sources & $14 \cdot 2^{\mathrm{a}}$ & 0.3 & $16 \cdot 4^{\mathrm{b}}$ & 0.4 & $17 \cdot 6^{\mathrm{c}}$ & 0.4 & $16 \cdot 7^{\mathrm{b}}$ & 0.4 \\
\hline Potatoes and other tubers & $3.5^{\mathrm{a}}$ & 0.1 & $3.5^{\mathrm{a}}$ & 0.2 & $4.4^{\mathrm{b}}$ & 0.1 & $4.4^{\mathrm{b}}$ & 0.2 \\
\hline Vegetables & $2 \cdot 1^{\mathrm{a}}$ & $0 \cdot 1$ & $2 \cdot 6^{\mathrm{b}}$ & 0.1 & $3 \cdot 1^{c}$ & $0 \cdot 1$ & $2 \cdot 6^{\mathrm{c}}$ & 0.1 \\
\hline Legumes & $0.097^{\mathrm{a}}$ & 0.029 & $0.255^{\mathrm{a}}$ & 0.073 & $0.220^{\mathrm{a}}$ & 0.060 & $0.137^{\mathrm{a}}$ & 0.044 \\
\hline \multicolumn{9}{|l|}{ Fruits } \\
\hline All fruit & $1.5^{\mathrm{a}}$ & 0.1 & $2 \cdot 5^{\mathrm{b}}$ & 0.2 & $3 \cdot 3^{c}$ & 0.2 & $3 \cdot 2^{\mathrm{c}}$ & 0.2 \\
\hline Fresh fruit & $1.4^{\mathrm{a}}$ & $0 \cdot 1$ & $2 \cdot 2^{\mathrm{b}}$ & 0.1 & $3 \cdot 1^{\mathrm{c}}$ & 0.2 & $3 \cdot 1^{\mathrm{c}}$ & 0.2 \\
\hline Nuts and seeds & $0.115^{\mathrm{a}}$ & 0.030 & $0.243^{b}$ & 0.051 & $0.207^{\mathrm{a}}$ & 0.057 & $0.083^{a}$ & 0.040 \\
\hline \multicolumn{9}{|l|}{ Cereals and cereal products } \\
\hline All & $7 \cdot 0^{\mathrm{a}}$ & 0.2 & $7 \cdot 6^{\mathrm{a}}$ & 0.3 & $6 \cdot 7^{\mathrm{a}}$ & 0.2 & $6 \cdot 4^{\mathrm{b}}$ & 0.2 \\
\hline Bread, crispbread and rusks & $5 \cdot 1^{\mathrm{a}}$ & 0.2 & $6 \cdot 4^{\mathrm{b}}$ & 0.3 & $6 \cdot 1^{b}$ & 0.2 & $5 \cdot 9^{\mathrm{b}}$ & 0.2 \\
\hline Other cereal products & $1.9^{\mathrm{a}}$ & $0 \cdot 1$ & $1 \cdot 2^{\mathrm{b}}$ & 0.1 & $0.557^{c}$ & 0.051 & $0.483^{c}$ & 0.063 \\
\hline \multicolumn{9}{|l|}{ Women } \\
\hline Total intake of main sources* & $11 \cdot 7^{\mathrm{a}}$ & 0.3 & $12 \cdot 9^{\mathrm{b}}$ & 0.3 & $14 \cdot 7^{\mathrm{C}}$ & 0.3 & $13.4^{\mathrm{b}}$ & 0.3 \\
\hline Potatoes and other tubers ${ }^{*}$ & $2 \cdot 2^{\mathrm{a}}$ & $0 \cdot 1$ & $2 \cdot 1^{\mathrm{a}}$ & 0.1 & $3 \cdot 0^{b}$ & $0 \cdot 1$ & $3 \cdot 2^{b}$ & $0 \cdot 1$ \\
\hline Vegetables & $1.9^{\mathrm{a}}$ & 0.1 & $2 \cdot 7^{\mathrm{b}}$ & 0.1 & $2 \cdot 9^{b}$ & 0.1 & $2 \cdot 5^{\mathrm{a}}$ & 0.1 \\
\hline Legumes & $0.060^{\mathrm{a}}$ & 0.019 & $0.141^{\mathrm{a}}$ & 0.043 & $0 \cdot 102^{\mathrm{a}}$ & 0.038 & $0.097^{\mathrm{a}}$ & 0.037 \\
\hline \multicolumn{9}{|l|}{ Fruits } \\
\hline All fruit* ${ }^{\star}$ & $2 \cdot 4^{\mathrm{a}}$ & $0 \cdot 1$ & $2 \cdot 7^{\mathrm{b}}$ & 0.1 & $3.7^{\mathrm{c}}$ & 0.2 & $3 \cdot 3^{\mathrm{c}}$ & 0.2 \\
\hline Fresh fruit* & $2 \cdot 2^{\mathrm{a}}$ & $0 \cdot 1$ & $2 \cdot 5^{\mathrm{b}}$ & 0.1 & $3 \cdot 6^{c}$ & 0.2 & $3 \cdot 3^{\mathrm{c}}$ & 0.2 \\
\hline Nuts and seeds & $0.191^{a}$ & 0.042 & $0.141^{a}$ & 0.030 & $0.094^{\mathrm{a}}$ & 0.023 & $0.065^{b}$ & 0.037 \\
\hline \multicolumn{9}{|l|}{ Cereals and cereal products } \\
\hline All ${ }^{*}$ & $5 \cdot 1^{a}$ & $0 \cdot 1$ & $5 \cdot 3^{\mathrm{a}}$ & 0.2 & $5 \cdot 0^{\mathrm{a}}$ & 0.2 & $4 \cdot 2^{b}$ & 0.1 \\
\hline Bread, crispbread and rusks* & $3 \cdot 7^{\mathrm{a}}$ & $0 \cdot 1$ & $4 \cdot 3^{\mathrm{b}}$ & 0.2 & $4 \cdot 5^{b}$ & $0 \cdot 1$ & $4 \cdot 0^{\mathrm{b}}$ & 0.1 \\
\hline Other cereal products & $1.5^{\mathrm{a} *}$ & 0.1 & $1 \cdot 0^{\mathrm{b}}$ & 0.1 & $0.466^{c}$ & 0.053 & $0.224^{d}$ & 0.029 \\
\hline
\end{tabular}

${ }_{a, b, c, d}$ Mean values within a row with unlike superscript letters were significantly different $(P<0 \cdot 05$, ANOVA with Bonferroni correction after log transformation). ${ }^{*}$ Mean values were significantly different between men and women $(P<0.05$, Student's $t$ test after log transformation). 
Table 4. Contribution ( $\mathrm{g} / \mathrm{d})$ of different food groups to the total fibre intake among the study population, stratified in sex-education groups (Mean values with their standard errors)

\begin{tabular}{|c|c|c|c|c|c|c|c|c|}
\hline \multirow[b]{3}{*}{ Food sources } & \multicolumn{8}{|c|}{ Education } \\
\hline & \multicolumn{2}{|c|}{$\begin{array}{l}\text { Lower secondary } \\
\quad \text { or less }\end{array}$} & \multicolumn{2}{|c|}{$\begin{array}{l}\text { Vocational, technical } \\
\text { or art education }\end{array}$} & \multicolumn{2}{|c|}{$\begin{array}{l}\text { General secondary } \\
\text { education }\end{array}$} & \multicolumn{2}{|c|}{ Higher education } \\
\hline & Mean & SEM & Mean & SEM & Mean & SEM & Mean & SEM \\
\hline \multicolumn{9}{|l|}{ Men } \\
\hline Total intake of main sources & $17 \cdot 6^{\mathrm{a}}$ & 0.4 & $15 \cdot 8^{\mathrm{b}}$ & 0.4 & $15 \cdot 3^{b}$ & 0.4 & $15 \cdot 9^{b}$ & 0.4 \\
\hline Potatoes and other tubers & $4 \cdot 7^{\mathrm{a}}$ & $0 \cdot 1$ & $4 \cdot 2^{b}$ & $0 \cdot 2$ & $3 \cdot 2^{\mathrm{c}}$ & 0.2 & $3 \cdot 3^{\mathrm{c}}$ & $0 \cdot 1$ \\
\hline Vegetables & $2 \cdot 7^{\mathrm{a}}$ & 0.1 & $2 \cdot 4^{\mathrm{b}}$ & 0.1 & $2 \cdot 3^{b}$ & 0.1 & $2 \cdot 8^{\mathrm{a}}$ & 0.1 \\
\hline Legumes & $0.240^{\mathrm{a}}$ & 0.069 & $0.149^{a}$ & 0.044 & $0 \cdot 127^{\mathrm{a}}$ & 0.042 & $0 \cdot 185^{\mathrm{a}}$ & 0.049 \\
\hline \multicolumn{9}{|l|}{ Fruits } \\
\hline All fruit & $2 \cdot 9^{\mathrm{a}}$ & 0.2 & $2 \cdot 1^{b}$ & 0.1 & $2 \cdot 3^{\mathrm{b}}$ & 0.2 & $3 \cdot 0^{\mathrm{a}}$ & 0.2 \\
\hline Fresh fruit & $2 \cdot 8^{\mathrm{a}}$ & 0.2 & $1.9^{b}$ & 0.1 & $2 \cdot 2^{b}$ & 0.2 & $2 \cdot 8^{\mathrm{a}}$ & 0.2 \\
\hline Nuts and seeds & $0.118^{\mathrm{a}}$ & 0.036 & $0.132^{\mathrm{a}}$ & 0.034 & $0 \cdot 140^{\mathrm{a}}$ & 0.038 & $0.236^{b}$ & 0.060 \\
\hline \multicolumn{9}{|l|}{ Cereals and cereal products } \\
\hline All & $7 \cdot 0^{\mathrm{a}}$ & 0.2 & $7 \cdot 1^{\mathrm{a}}$ & 0.2 & $7 \cdot 3^{\mathrm{a}}$ & 0.3 & $6 \cdot 6^{\mathrm{a}}$ & 0.2 \\
\hline Bread, crispbread and rusks & $6 \cdot 4^{\mathrm{a}}$ & 0.2 & $6 \cdot 0^{\mathrm{a}}$ & 0.2 & $5 \cdot 6^{b}$ & 0.2 & $5 \cdot 4^{b}$ & 0.2 \\
\hline Other cereal products & $0.560^{\mathrm{a}}$ & 0.056 & $1 \cdot 1^{\mathrm{b}}$ & 0.1 & $1 \cdot 7^{\mathrm{C}}$ & 0.1 & $1 \cdot 1^{b}$ & 0.1 \\
\hline \multicolumn{9}{|l|}{ Women } \\
\hline Total intake of main sources ${ }^{*}$ & $13 \cdot 5^{a}$ & 0.3 & $12 \cdot 2^{\mathrm{b}}$ & 0.3 & $13 \cdot 2^{\mathrm{a}}$ & 0.4 & $13 \cdot 6^{\mathrm{a}}$ & 0.3 \\
\hline Potatoes and other tubers* & $3 \cdot 2^{\mathrm{a}}$ & 0.1 & $2 \cdot 5^{\mathrm{b}}$ & 0.1 & $2 \cdot 5^{\mathrm{b}}$ & 0.1 & $2 \cdot 1^{\mathrm{c}}$ & 0.1 \\
\hline Vegetables & $2 \cdot 5^{\mathrm{a}}$ & 0.1 & $2 \cdot 2^{b}$ & 0.1 & $2 \cdot 5^{\mathrm{a}}$ & 0.1 & $3 \cdot 0^{c}$ & 0.1 \\
\hline Legumes & $0 \cdot 135^{\mathrm{a}}$ & 0.038 & $0 \cdot 108^{\mathrm{a}}$ & 0.049 & $0 \cdot 100^{\mathrm{a}}$ & 0.031 & $0.036^{a}$ & 0.019 \\
\hline \multicolumn{9}{|l|}{ Fruits } \\
\hline All fruit* & $2 \cdot 9^{\mathrm{a}}$ & 0.1 & $2 \cdot 8^{\mathrm{a}}$ & 0.2 & $3 \cdot 2^{\mathrm{a}}$ & 0.2 & $3 \cdot 2^{\mathrm{a}}$ & 0.2 \\
\hline Fresh fruit* & $2 \cdot 9^{\mathrm{a}}$ & 0.1 & $2 \cdot 6^{\mathrm{b}}$ & 0.2 & $3.0^{\mathrm{a}}$ & 0.2 & $3.0^{\mathrm{a}}$ & 0.2 \\
\hline Nuts and seeds & $0.040^{\mathrm{a}}$ & 0.016 & $0.150^{\mathrm{a}}$ & 0.037 & $0 \cdot 191^{\mathrm{b}}$ & 0.046 & $0 \cdot 177^{\mathrm{a}}$ & 0.044 \\
\hline \multicolumn{9}{|l|}{ Cereals and cereal products } \\
\hline All & $4 \cdot 8^{\mathrm{a}}$ & 0.1 & $4 \cdot 7^{\mathrm{a}}$ & 0.2 & $5 \cdot 0^{\mathrm{a}}$ & 0.2 & $5 \cdot 3^{b}$ & 0.2 \\
\hline Bread, crispbread and rusks* & $4 \cdot 4^{\mathrm{a}}$ & 0.1 & $3 \cdot 7^{b}$ & 0.1 & $3.9^{b}$ & 0.2 & $4 \cdot 3^{\mathrm{a}}$ & 0.2 \\
\hline Other cereal products ${ }^{*}$ & $0.403^{\mathrm{a}}$ & 0.104 & $1.0^{\mathrm{b}}$ & 0.1 & $1 \cdot 1^{b}$ & 0.1 & $0.925^{\mathrm{b}}$ & 0.065 \\
\hline
\end{tabular}

a,b,c Mean values within a row with unlike superscript letters were significantly different $(P<0.05$, ANOVA with Bonferroni correction after log transformation). * Mean values were significantly different between men and women $(P<0.05$; Student's $t$ test after log transformation).

The dietary fibre intakes of the Belgian population were comparable to the European levels (male adolescents, 14.0$26 \mathrm{~g} / \mathrm{d}$; female adolescents, $14.0-22 \mathrm{~g} / \mathrm{d}$; male adults, 18.0 $26 \mathrm{~g} / \mathrm{d}$; female adults, $16 \cdot 0-26 \mathrm{~g} / \mathrm{d}$; elderly men, $15 \cdot 0-31 \mathrm{~g} / \mathrm{d}$, and elderly women, $16 \cdot 0-23 \mathrm{~g} / \mathrm{d})^{(40)}$. Belgian men had similar dietary fibre intakes as Catalan men in Spain using the same method in 10- to 75-year-old subjects, while Belgian women in all age groups consumed substantially less dietary fibres than Spanish women (Spanish $17.0 \mathrm{~g} / \mathrm{d})^{(41)}$. Additionally, Spanish male adolescents (mean $18.7 \mathrm{~g} / \mathrm{d}$ ) had slightly higher dietary fibre intakes than Belgian male adolescents $(17.8 \mathrm{~g} / \mathrm{d})$, whereas Belgian adolescents had slightly higher dietary fibre intakes than Italian adolescents via $24 \mathrm{~h}$ dietary records (Belgium: men, $17.8 \mathrm{~g} / \mathrm{d}$; women, $15.0 \mathrm{~g} / \mathrm{d}$; Italy: men, $17.0 \mathrm{~g} / \mathrm{d}$; women, $14.0 \mathrm{~g} / \mathrm{d})^{(42)}$.

According to two American national nutrition surveys using $24 \mathrm{~h}$ recalls (1988-91: all age categories; 1988-2004: adults), lower total fibre intakes were reported among the US population $(14 \cdot 0-16 \cdot 7 \text { and } 16 \cdot 0-19 \cdot 0 \mathrm{~g} / \mathrm{d} \text {, respectively })^{(43,44)}$. Furthermore, based on a Japanese dietary survey among the general population, Japanese and Belgian men had similar fibre intakes, whereas Japanese women $(14 \cdot 7-21 \mathrm{~g} / \mathrm{d})$ consumed comparatively more fibres than Belgian women in all age groups ${ }^{(8)}$. However, Japanese men $<30$ years $(13.7 \mathrm{~g} / \mathrm{d})$ consumed a substantially lower amount of fibres compared with the same Belgian population group $(18 \cdot 1 \mathrm{~g} / \mathrm{d})$.
In the present study, the most important contributors to dietary fibre intake were cereals and cereal products (bread, crispbread and rusks in particular), followed by potatoes and other tubers, vegetables and fruits (mainly fresh fruits). Similar food groups were identified as the major contributors in Italian adolescents ${ }^{(42)}$. Results of the Spanish and Japanese national surveys, on the other hand, indicate that potatoderived fibre intakes contributed much more to the Belgian $(14.4 \%, 3.3 \mathrm{~g} / \mathrm{d})$ than to Spanish and Japanese total fibre intakes $(6.0 \%$, approximately $1.1 \mathrm{~g} / \mathrm{d}$ and approximately $1.5 \mathrm{~g} / \mathrm{d}$, respectively $)^{(8,41)}$. While the contribution of cerealderived fibres was higher for Belgians than for Spanish persons $(34 \%, 5.9 \mathrm{~g} / \mathrm{d} v .28 \%$, approximately $5.2 \mathrm{~g} / \mathrm{d})$, the contribution of fruit-derived fibres was considerably lower in Belgians compared with Spanish $(14.7 \%, 2.8 \mathrm{~g} / \mathrm{d} v .22 \%$, approximately $3.9 \mathrm{~g} / \mathrm{d}$ ). Additionally, the contribution of fruit-derived fibres in the Belgian was similar to that in the Japanese population $(13.5 \%, 2.5 \mathrm{~g} / \mathrm{d})$. The fibre intakes from the remaining food groups, including legumes, nuts and seeds, and vegetables, were lower in this Belgium sample than in the other surveys. In particular, the consumption and contribution of legume-derived fibres were extremely low compared with Spanish $(12 \cdot 1 \% \text {, approximately } 2 \cdot 2 \mathrm{~g} / \mathrm{d})^{(41)}$ and Japanese $(14 \cdot 1 \% \text {, approximately } 2.6 \mathrm{~g} / \mathrm{d})^{(8)}$ reports.

To the best of our knowledge, no published Belgian data are available describing dietary fibre intakes stratified by 
Table 5. Stepwise multiple linear regression analysis of the potential associations between BMI and waist circumference (WC), and total and food group-specific fibre intakes among participants of the Belgian national food consumption survey (2004-5)

(Coefficients with their standard errors and $95 \%$ confidence intervals)

\begin{tabular}{|c|c|c|c|c|}
\hline & $\beta$ Coefficient & SE & $95 \% \mathrm{Cl}$ & $P$ \\
\hline \multirow{2}{*}{\multicolumn{5}{|c|}{ Dependent variable: $\mathrm{BMI}\left(\mathrm{kg} / \mathrm{m}^{2} ; n 3055\right)^{\star} \dagger \ddagger$}} \\
\hline \multicolumn{3}{|l|}{ Food groups§ } & & \\
\hline \multicolumn{5}{|c|}{ Potatoes and other tubers } \\
\hline Model 1 & 0.139 & 0.029 & $0.081,0.197$ & $<0.001$ \\
\hline Model 2 & 0.022 & 0.023 & $-0.024,0.067$ & 0.353 \\
\hline Model 3 & -0.009 & 0.028 & $-0.064,0.046$ & 0.754 \\
\hline \multicolumn{5}{|l|}{ Vegetables } \\
\hline Model 1 & 0.112 & 0.037 & $0.039,0.186$ & 0.003 \\
\hline Model 2 & 0.065 & 0.034 & $-0.002,0.131$ & 0.057 \\
\hline Model 3 & 0.056 & 0.034 & $-0.010,0.123$ & 0.095 \\
\hline \multicolumn{5}{|l|}{ Fruits } \\
\hline Model 1 & 0.107 & 0.025 & $0.058,0.157$ & $<0.001$ \\
\hline Model 2 & 0.022 & 0.023 & $-0.024,0.067$ & 0.353 \\
\hline Model 3 & 0.024 & 0.023 & $-0.021,0.069$ & 0.297 \\
\hline \multicolumn{5}{|c|}{ Cereals and cereal products } \\
\hline Model 1 & -0.077 & 0.020 & $-0.118,-0.037$ & $<0.001$ \\
\hline Model 2 & -0.045 & 0.020 & $-0.084,-0.006$ & 0.025 \\
\hline Model 3 & -0.019 & 0.022 & $-0.063,0.025$ & 0.391 \\
\hline \multicolumn{5}{|c|}{ Dependent variable: WC $(\mathrm{cm} ; n 2875)^{\star} \dagger \ddagger$} \\
\hline \multicolumn{5}{|l|}{ Total fibre\| } \\
\hline Model 1 & 0.172 & 0.037 & $0.099,0.244$ & $<0.001$ \\
\hline Model 2 & -0.091 & 0.030 & $-0.150,-0.032$ & 0.003 \\
\hline Model 3 & -0.118 & 0.032 & $-0.181,-0.055$ & $<0.001$ \\
\hline \multicolumn{5}{|l|}{ Food groups } \\
\hline \multicolumn{5}{|c|}{ Potatoes and other tubers } \\
\hline Model 1 & 0.876 & 0.102 & $0.676,1.076$ & $<0.001$ \\
\hline Model 2 & -0.003 & 0.086 & $-0.171,0.165$ & 0.972 \\
\hline Model 3 & -0.028 & 0.085 & $-0.195,0.140$ & 0.744 \\
\hline \multicolumn{5}{|l|}{ Vegetables } \\
\hline Model 1 & 0.288 & 0.131 & $0.031,0.545$ & 0.028 \\
\hline Model 2 & 0.063 & 0.105 & $-0.143,0.269$ & 0.548 \\
\hline Model 3 & 0.099 & 0.104 & $-0.105,0.303$ & 0.342 \\
\hline \multicolumn{5}{|l|}{ Fruits } \\
\hline Model 1 & 0.334 & 0.087 & $0.164,0.505$ & $<0.001$ \\
\hline Model 2 & -0.066 & 0.070 & $-0.204,0.072$ & 0.349 \\
\hline Model 3 & 0.731 & 0.212 & $0.316,1.146$ & 0.001 \\
\hline
\end{tabular}

${ }^{*}$ Model 1, unadjusted; model 2, adjusted for age, sex, region and level of education; model 3 , model 2 further adjusted for total energy intake and interactions with and between the effect factors.

t Legume-derived fibre intake was not retained in the models for both BMI and WC, whereas total fibre intakes and cereal-derived fibre intakes were not retained in the model for $\mathrm{BMI}$ and $\mathrm{WC}$, respectively.

$\ddagger$ Women, lower secondary education and Walloon region are as reference in the models.

$\S$ Model 2. Significantly positively associated variable: age; significantly negatively associated variables: sex, general secondary education, higher education, Flanders region and Brussels region. Model 3 . Significantly positively associated variables: age, sex, higher education and Brussels region; significantly negatively associated variables: general secondary education and Flanders region. Significantly positively associated interactions: vocational, technical or art $\times$ sex, higher education $\times$ age and Brussels region $\times$ age; significantly negatively associated interactions: sex $\times$ age, vocational, technical or art $\times$ age, general secondary education $\times$ sex, general secondary education $\times$ age, higher education $\times \operatorname{sex}$, and cereal fibre $\times$ vocational, technical or art.

\| Model 2. Significantly positively associated variable: age; significantly negatively associated variables: sex, general secondary education and Brussels region. Mode 3. Significantly positively associated variables: age and Brussels region; significantly negatively associated variables: sex and general secondary education. Significantly positively associated interactions: general secondary education $\times$ age and total fibre $\times$ higher education; significantly negatively associated interactions: $\operatorname{sex} \times$ age, higher education $\times$ sex and Brussels region $\times$ age.

q Model 2. Significantly positively associated variable: age; significantly negatively associated variables: sex, general secondary education and higher education. Model 3. Significantly positively associated variables: age and higher education; significantly negatively associated variables: sex and general secondary education. Significantly positively associated interaction: general secondary education $\times$ age; significantly negatively associated interactions: higher education $\times$ age, Brussels region $\times$ age, fruit fibre $\times$ sex, cereal fibre $\times$ vocational, technical or art, and cereal fibre $\times$ general secondary education. level of education. There are indications that a higher socioeconomic status such as education, family income and occupational level is associated with higher intakes of dietary fibres ${ }^{(45-49)}$. Nevertheless, in our Belgian study, lower secondary educated men and higher educated women reported the highest total fibre intakes. In all sex-education groups, cereals and cereal products contributed most to the total fibre intake. The contribution of cereal products other than bread, crispbread or rusks was the lowest for lower secondary educated men and women, whereas higher educated persons consumed more fibres from vegetables and fruits. In general, potato-derived fibre intakes decreased with the level of education. Moreover, some recent studies have observed that fibre intake through the consumption of vegetables, fruits and cereals increases with participants' socio-economic status and education level ${ }^{(45,48)}$.

\section{Associations between dietary fibre intake and BMI and waist circumference}

Although BMI is not an accurate indicator of body composition, it is a well-known predictor of obesity in the general population $^{(50)}$. WC is a better and stronger predictor of abdominal obesity and obesity-related health risks ${ }^{(51)}$. Hence, in the present study, both BMI and WC were used in order to find more precise associations between dietary fibre intake and body composition.

There are indications that the consumption of dietary fibres may have beneficial effects, such as lowering body weight, $\mathrm{BMI}$ and $\mathrm{WC}^{(52-62)}$. In the present study, total dietary fibre intakes were significantly inversely associated with WC, but not with BMI. In line with the present findings, Ventura et $a l .{ }^{(62)}$ reported that higher total dietary fibre intakes correlated with lower WC. Moreover, Liese et al. ${ }^{(56)}$ found that total fibre intakes were inversely associated with both BMI $(\beta=-0.795, P=0.013)$ and WC $(\beta=-1.9, P=0.008)$ after adjustment for age, sex and socio-economic status. Du et $a l .{ }^{(63)}$ observed that dietary fibre intakes were inversely associated with body-weight gain and WC increases among Europeans. Conversely, one cross-sectional study involving adults aged $60-80$ years and one randomised controlled trial involving breast cancer patients aged 18-70 years reported no significant effects of dietary fibres on BMI or body weight $^{(57,60)}$. Additionally, in one recent cross-sectional study involving 5783 Chinese adults aged 20-59 years, higher total dietary fibre intakes correlated with increased $\mathrm{BMI}^{(59)}$.

Although cereal-derived fibre intake was the most important contributor in all sex-age and sex-education groups in the present study, it was not significantly associated with BMI nor WC. A European prospective cohort study ${ }^{(63)}$, however, suggested that fibres derived from cereals, more than those from vegetables or fruits, may have a role in the prevention of body-weight and WC gain. In addition, recent studies have found that cereal-derived fibre intakes were inversely associated with $\mathrm{BMI}$ and $\mathrm{WC}^{(9,16,57,64)}$. A recent systematic review has suggested that the consumption of cereal fibre led to more health benefits in the prevention of type 2 diabetes mellitus by improving insulin sensitivity and increased 
bowel movements without adverse effects ${ }^{(65)}$. Furthermore, one prospective cohort study and one cross-over study have stated that a high cereal fibre intake with a low glycaemic load was inversely associated with the risk of type 2 diabetes compared with lower cereal fibre intake and higher glycaemic $\operatorname{load}^{(66,67)}$. Surprisingly, in the present study, the intake of fruit-derived fibres was positively associated with WC. Conversely, McKeown et al. ${ }^{(57)}$ found no significant associations between the intake of fibres from vegetables or fruits and body composition. Moreover, some recent studies have described an inverse relationship between fruit- and vegetable-derived fibre intakes and body weight and $\mathrm{BMI}^{(68,69)}$. According to recent reviews, high fibre intakes through the consumption of vegetables and fruits would have no direct effect on body weight, but may exert indirectly health-promoting activities related to body composition ${ }^{(70,71)}$.

In the present study, we controlled for potential modifier effects and observed that age, sex, region, education, energy intake and certain interactions affected the linear association between dietary fibre intakes and BMI and WC. Similarly, region, age, sex and level of education have been described as potential confounding factors for the effect of fibre intake on measures of obesity ${ }^{(52)}$. Byrd-Williams et al. ${ }^{(53)}$ found that dietary fibre intakes were inversely associated with the BMI of men and women (18-24 years), and with the WC of men. Howarth et al. ${ }^{(55)}$, on the other hand, reported that dietary fibre intakes were inversely associated with the BMI of women aged 20-59 years, but not of men in the same age range.

\section{Strengths and limitations}

This national nutrition survey can be seen as the largest survey covering all the provinces and language regions in Belgium so far. The present study is the first one evaluating dietary fibre intake stratified by sex-age and sex-education, as well as assessing the association with anthropometric indices including BMI and $\mathrm{WC}$ in the Belgian population.

Yet, some limitations of the present study are the low response rate (42\%) and the use of two $24 \mathrm{~h}$ recalls to assess dietary fibre intakes. Although, no doubt, willingness to participate leads to some selection bias as volunteers are generally more concerned with health and diet than others, the present study population represents a more general population of Belgium in comparison with other studies, which are mostly restricted to local areas. In addition, all seasons and days of the week were almost equally represented. Another limitation related to the sampling is the broad age range of the young adult group (19-59 years), which could lead to imprecise interpretations of the results obtained for that age group. Differences attributed to this age group may be applicable only to a smaller subset with more significant differences, and this may have implications for policy recommendations

A limitation of $24 \mathrm{~h}$ dietary recalls is that it does not allow quantifying proportions of non-consumers for particular food items, a fortiori for infrequently consumed foods. Moreover, accuracy of collected data relies on the individual's ability to remember foods and beverages consumed in the past $24 \mathrm{~h}$, and might, therefore, be biased towards misreporting. In this respect, the $24 \mathrm{~h}$ dietary recalls were performed through computer-assisted EPIC-SOFT and face-to-face interviews to guide the participants to report all their consumption, even the easily forgotten snacks.

Another limitation of this survey is the fact that weight and height were self-reported. However, the strength of this survey is that WC was measured by trained dietitians. When interpreting the results, it could be that this difference in recording method (self-reported $v$. measured) is partly responsible for the differences found between BMI and WC in the present study. Yet, the inclusion of both measures is an important strength of the present study, as these are not similar but complementary parameters for body composition due to their independent contribution to the prediction of total and abdominal obesity ${ }^{(72)}$.

Additionally, a large number of missing values of WC might be a factor leading to a biased sample, as obese people might more often refuse to have a WC measurement. Then, the underestimated WC could bias the association between WC and dietary fibre intake in the present study. However, the authors performed some additional sensitivity analyses (results not shown), which showed no significant differences in BMI or fibre intake between the group with missing WC data and those included in the WC analyses.

At last, it is noteworthy that soluble and insoluble dietary fibre intake should be analysed separately for further knowledge, but due to the information in the food composition databases, this was not possible. Biomarkers as well need to be considered to investigate the association with dietary fibre intake and glycaemic index in future studies.

\section{Recommendations}

The present study findings indicate unfavourably low fibre intakes among our Belgian population. Enhancing the daily amount of vegetables, fruits, legumes, nuts and seeds could contribute importantly to higher fibre intakes. However, in the battle against obesity, one should keep in mind that the total energy intake should not be influenced by the enhancement of these food groups, which could necessitate a concomitant decrease in other (less fibre-rich and energy-dense) foods such as soft drinks, candies and refined bakery products. If public health policies for the increase in fibre intakes are to be effective, policy development and implementation need to target the main sources of dietary fibre in various populations. Also manufacturers could be stimulated to reformulate existing products to incorporate more whole grains.

\section{Conclusion}

This survey provides information on the consumption of total and food group-specific fibres in Belgium. The most important contributor was cereals and cereal products. Total fibre intakes were lower than the recommended intake in all sex-age and sex-education groups. The dietary fibre intakes increased with age. Lower educated men and higher educated women 
consumed most dietary fibre. Although these results show that Belgians did not consume enough fibre, a significant inverse relationship was observed between total fibre and cerealderived fibre intakes and WC.

\section{Acknowledgements}

The present study was supported by the Alpro Foundation. The food consumption survey was funded by the Federal Ministry of Health, Food Chain Safety and Environment. Y. L. performed and interpreted the statistical analyses and drafted the manuscript. A. P. and M. D. N., the dietitians of our team, were responsible for the data linking with the food composition databases. S. D. V., H. V. O. and I. H. were responsible for the coordination of the fieldwork of the Belgian food consumption survey, and G. D. B. and S. D. H. were involved in the conceptualisation of the national food consumption survey. All other authors helped in the evaluation of the results and the writing of the manuscript. All authors have evaluated and approved the final version of the manuscript. The authors declare that there are no conflicts of interests.

\section{References}

1. Cummings JH, Mann JI, Nishida C, et al. (2009) Dietary fibre: an agreed definition. Lancet 373, 365-366.

2. Rees G, Davies J, Thompson R, et al. (2005) Randomisedcontrolled trial of a fibre supplement on the symptoms of irritable bowel syndrome. J R Soc Promot Health 125, 30-34.

3. Tan KY \& Seow-Choen F (2007) Fiber and colorectal diseases: separating fact from fiction. World J Gastroenterol 13, 4161-4167.

4. Tarini J \& Wolever TM (2010) The fermentable fibre inulin increases postprandial serum short-chain fatty acids and reduces free-fatty acids and ghrelin in healthy subjects. Appl Physiol Nutr Metab 35, 9-16.

5. Aleixandre A \& Miguel M (2008) Dietary fibre in the prevention and treatment of metabolic syndrome: a review. Crit Rev Food Sci Nutr 48, 905-912.

6. Mathern JR, Raatz SK, Thomas W, et al. (2009) Effect of fenugreek fiber on satiety, blood glucose and insulin response and energy intake in obese subjects. Phytother Res 23, $1543-1548$.

7. Chandalia M, Garg A, Lutjohann D, et al. (2000) Beneficial effects of high dietary fibre intake in patients with type 2 diabetes mellitus. $N$ Engl J Med 342, 1392-1398.

8. Fukuda S, Saito H, Nakaji S, et al. (2007) Pattern of dietary fibre intake among the Japanese general population. Eur $J$ Clin Nutr 61, 99-103.

9. Newby PK, Maras J, Bakun P, et al. (2007) Intake of whole grains, refined grains, and cereal fibre measured with 7-d diet records and associations with risk factors for chronic disease. Am J Clin Nutr 86, 1745-1753.

10. Anderson JW, Baird P, Davis RH, et al. (2009) Health benefits of dietary fiber. Nutr Rev 67, 188-205.

11. Ludwig DS (2002) The glycemic index: physiological mechanisms relating to obesity, diabetes, and cardiovascular disease. JAMA 287, 2414-2423.

12. Davis JN, Hodges VA \& Gillham MB (2006) Normal-weight adults consume more fiber and fruit than their age- and height-matched overweight/obese counterparts. J Am Diet Assoc 106, 833-840.
13. Deshmukh-Taskar PR, Nicklas TA, O'Neil CE, et al. (2010) The relationship of breakfast skipping and type of breakfast consumption with nutrient intake and weight status in children and adolescents: the National Health and Nutrition Examination Survey 1999-2006. J Am Diet Assoc 110 869-878.

14. Krishnan S, Rosenberg L, Singer M, et al. (2007) Glycemic index, glycemic load, and cereal fibre intake and risk of type 2 diabetes in US black women. Arch Intern Med 167, 2304-2309.

15. Ventura E, Davis J, Byrd-Williams C, et al. (2009) Reduction in risk factors for type 2 diabetes mellitus in response to a low-sugar, high-fiber dietary intervention in overweight Latino adolescents. Arch Pediatr Adolesc Med 163, $320-327$.

16. McKeown NM, Meigs JB, Liu S, et al. (2004) Carbohydrate nutrition, insulin resistance, and the prevalence of the metabolic syndrome in the Framingham Offspring Cohort. Diabetes Care 27, 538-546.

17. Federal Public Service for Public Health \& Food-Chain Safety and Environment (2006) Dietary recommendations for Belgium. https://portal.health.fgov.be/portal/page?_pageid=56, $4190386 \&$ dad=portal\&_schema=PORTAL\&_menu=menu_ 3_1 (accessed March 2010).

18. World Health Organization (2009) Population nutrient intake goals for preventing diet-related chronic diseases. http:// www.who.int/dietphysicalactivity/publications/trs916/en/ gsfao_overall.pdf (accessed December 2009).

19. World Health Organization (WHO) (1998) Preparation and Use of Food-based Dietary Guidelines. Report of a Joint FAO/WHO Consultation. 880, pp. i-108. Geneva: WHO.

20. Vandevijvere S, De Vriese S, Huybrechts I, et al. (2009) The gap between food-based dietary guidelines and usual food consumption in Belgium, 2004. Public Health Nutr 12, 423-431.

21. Huybrechts I \& De Henauw S (2007) Energy and nutrient intakes by pre-school children in Flanders-Belgium. $\mathrm{Br} J$ Nutr 98, 600-610.

22. Matthys C, De Henauw S, Devos C, et al. (2003) Estimated energy intake, macronutrient intake and meal pattern of Flemish adolescents. Eur J Clin Nutr 57, 366-375.

23. Charafeddine R, Van Oyen H \& Demarest S (2009) Trends in social inequalities in obesity: Belgium, 1997 to 2004. Prev Med 48, 54-58.

24. Stam-Moraga MC, Kolanowski J, Dramaix M, et al. (1999) Sociodemographic and nutritional determinants of obesity in Belgium. Int J Obes Relat Metab Disord 23, Suppl. 1, 1-9.

25. De Vriese S, De Backer G, De Henauw S, et al. (2005) The Belgian food consumption survey: aims, design and methods. Arch Public Health 63, 1-16.

26. Brussaard JH, Johansson L \& Kearney J (2002) Rationale and methods of the EFCOSUM project. Eur J Clin Nutr 56, Suppl. 2, S4-S7.

27. Slimani N \& Valsta L (2002) Perspectives of using the EPICSOFT programme in the context of pan-European nutritional monitoring surveys: methodological and practical implications. Eur J Clin Nutr 56, Suppl. 2, S63-S74.

28. Butler RC \& Patel J (2000) A direction in dietary fibre analysis. Nutr Food Sci 30, 221-226.

29. NUBEL (2004) Belgium Food Composition Table, 4th ed Brussel: Ministry of Public Health (in Dutch).

30. NEVO (2003) NEVO-Table, Dutch Food Composition Table 2003. Zeist: NEVO Foundation (in Dutch).

31. US Department of Agriculture (USDA) \& US Department of Health and Human Services (2005) Dietary Guidelines for 
Americans. www.healthierus.gov/dietaryguidelines (accessed December 2009).

32. World Health Organization (2007) Body mass index (BMI). http://www.euro.who.int/nutrition/20030507_1 (accessed December 2009).

33. Cole TJ, Bellizzi MC, Flegal KM, et al. (2000) Establishing a standard definition for child overweight and obesity worldwide: international survey. Br Med J 320, 1240-1243.

34. Vrije Universiteit Brussel, Antropogenetica, Katholieke Universiteit Leuven, \& Jeugdgezondheidszorg (2004) Growth Charts, Flanders 2004. http://www.vub.ac.be/groeicurven/ english.html (accessed December 2009).

35. Lean ME, Han TS \& Morrison CE (1995) Waist circumference as a measure for indicating need for weight management. Br Med J 311, 158-161.

36. Taylor RW, Jones IE, Williams SM, et al. (2000) Evaluation of waist circumference, waist-to-hip ratio, and the conicity index as screening tools for high trunk fat mass, as measured by dual-energy X-ray absorptiometry, in children aged 3-19y. Am J Clin Nutr 72, 490-495.

37. Trumbo P, Schlicker S, Yates AA, et al. (2002) Dietary reference intakes for energy, carbohydrate, fiber, fat, fatty acids, cholesterol, protein and amino acids. J Am Diet Assoc 102, 1621-1630.

38. British Nutrition Foundation (2010) Dietary fibre. http://www. nutrition.org.uk/nutritionscience/nutrients/dietary-fibre?start $=4$ (accessed January 2010).

39. Williams CL, Bollella M \& Wynder EL (1995) A new recommendation for dietary fiber in childhood. Pediatrics $\mathbf{9 6}$, 985-988.

40. Elmadfa I \& Freisling H (2009) Nutritional status in Europe: methods and results. Nutr Rev 67, Suppl. 1, S130-S134.

41. Serra-Majem L, Ribas-Barba L, Salvador G, et al. (2007) Trends in energy and nutrient intake and risk of inadequate intakes in Catalonia, Spain (1992-2003). Public Health Nutr 10, 1354-1367.

42. Leclercq C, Piccinelli R, Arcella D, et al. (2004) Food consumption and nutrient intake in a sample of Italian secondary school students: results from the INRAN-RM-2001 food survey. Int J Food Sci Nutr 55, 265-277.

43. Alaimo K, McDowell MA, Briefel RR, et al. (1994) Dietary intake of vitamins, minerals, and fiber of persons ages 2 months and over in the United States: Third National Health and Nutrition Examination Survey, Phase 1, 1988-91. Adv Data $1-28$.

44. Oza-Frank R, Cheng YJ, Narayan KM, et al. (2009) Trends in nutrient intake among adults with diabetes in the United States: 1988-2004. J Am Diet Assoc 109, 1173-1178.

45. Bhargava A (2004) Socio-economic and behavioural factors are predictors of food use in the National Food Stamp Program Survey. Br J Nutr 92, 497-506.

46. Hjartaker A \& Lund E (1998) Relationship between dietary habits, age, lifestyle, and socio-economic status among adult Norwegian women. The Norwegian Women and Cancer Study. Eur J Clin Nutr 52, 565-572.

47. Hulshof KF, Brussaard JH, Kruizinga AG, et al. (2003) Socioeconomic status, dietary intake and $10 \mathrm{y}$ trends: the Dutch National Food Consumption Survey. Eur J Clin Nutr 57, $128-137$.

48. Lang R \& Jebb SA (2003) Who consumes whole grains, and how much? Proc Nutr Soc 62, 123-127.

49. Zhang H, Hsu-Hage BH \& Wahlqvist ML (2002) Longitudinal changes in nutrient intakes in the Melbourne Chinese Cohort Study. Public Health Nutr 5, 433-439.
50. Mafra D, Guebre-Egziabher F \& Fouque D (2008) Body mass index, muscle and fat in chronic kidney disease: questions about survival. Nephrol Dial Transplant 23, 2461-2466.

51. Chan DC, Watts GF, Barrett PH, et al. (2003) Waist circumference, waist-to-hip ratio and body mass index as predictors of adipose tissue compartments in men. QJM 96, $441-447$.

52. Affenito SG, Thompson DR, Barton BA, et al. (2005) Breakfast consumption by African-American and white adolescent girls correlates positively with calcium and fibre intake and negatively with body mass index. J Am Diet Assoc 105, 938-945.

53. Byrd-Williams CE, Strother ML, Kelly LA, et al. (2009) Dietary fibre and associations with adiposity and fasting insulin among college students with plausible dietary reports. Nutrition 25, 896-904.

54. Duvigneaud N, Wijndaele K, Matton L, et al. (2007) Dietary factors associated with obesity indicators and level of sports participation in Flemish adults: a cross-sectional study. Nutr J 6, 26.

55. Howarth NC, Huang TT, Roberts SB, et al. (2005) Dietary fibre and fat are associated with excess weight in young and middle-aged US adults. J Am Diet Assoc 105, $1365-1372$.

56. Liese AD, Schulz M, Fang F, et al. (2005) Dietary glycemic index and glycemic load, carbohydrate and fiber intake, and measures of insulin sensitivity, secretion, and adiposity in the Insulin Resistance Atherosclerosis Study. Diabetes Care 28, 2832-2838.

57. McKeown NM, Yoshida M, Shea MK, et al. (2009) Wholegrain intake and cereal fibre are associated with lower abdominal adiposity in older adults. J Nutr 139, 1950-1955.

58. Murakami K, Sasaki S, Okubo H, et al. (2007) Dietary fibre intake, dietary glycemic index and load, and body mass index: a cross-sectional study of 3931 Japanese women aged 18-20 years. Eur J Clin Nutr 61, 986-995.

59. Stookey JD (2001) Energy density, energy intake and weight status in a large free-living sample of Chinese adults: exploring the underlying roles of fat, protein, carbohydrate, fiber and water intakes. Eur J Clin Nutr 55, 349-359.

60. Thomson CA, Rock CL, Giuliano AR, et al. (2005) Longitudinal changes in body weight and body composition among women previously treated for breast cancer consuming a high-vegetable, fruit and fiber, low-fat diet. Eur J Nutr $\mathbf{4 4}$, $18-25$.

61. Tucker LA \& Thomas KS (2009) Increasing total fiber intake reduces risk of weight and fat gains in women. J Nutr 139, $576-581$.

62. Ventura EE, Davis JN, Alexander KE, et al. (2008) Dietary intake and the metabolic syndrome in overweight Latino children. J Am Diet Assoc 108, 1355-1359.

63. Du H, van der ADL, Boshuizen HC, et al. (2009) Dietary fibre and subsequent changes in body weight and waist circumference in European men and women. Am J Clin Nutr 91, 329-336.

64. Good CK, Holschuh N, Albertson AM, et al. (2008) Whole grain consumption and body mass index in adult women: an analysis of NHANES 1999-2000 and the USDA pyramid servings database. J Am Coll Nutr 27, 80-87.

65. Park Y, Brinton LA, Subar AF, et al. (2009) Dietary fibre intake and risk of breast cancer in postmenopausal women: the National Institutes of Health-AARP Diet and Health Study. Am J Clin Nutr 90, 664-671.

66. Fung TT, Hu FB, Pereira MA, et al. (2002) Whole-grain intake and the risk of type 2 diabetes: a prospective study in men. Am J Clin Nutr 76, 535-540. 
67. Samra RA \& Anderson GH (2007) Insoluble cereal fiber reduces appetite and short-term food intake and glycemic response to food consumed $75 \mathrm{~min}$ later by healthy men. Am J Clin Nutr 86, 972-979.

68. Koh-Banerjee P, Franz M, Sampson L, et al. (2004) Changes in whole-grain, bran, and cereal fiber consumption in relation to 8-y weight gain among men. Am J Clin Nutr 80, $1237-1245$.

69. Sartorelli DS, Franco LJ \& Cardoso MA (2008) High intake of fruits and vegetables predicts weight loss in Brazilian overweight adults. Nutr Res 28, 233-238.
70. Feldeisen SE \& Tucker KL (2007) Nutritional strategies in the prevention and treatment of metabolic syndrome. Appl Physiol Nutr Metab 32, 46-60.

71. Kaline K, Bornstein SR, Bergmann A, et al. (2007) The importance and effect of dietary fibre in diabetes prevention with particular consideration of whole grain products. Horm Metab Res 39, 687-693.

72. Janssen I, Heymsfield SB, Allison DB, et al. (2002) Body mass index and waist circumference independently contribute to the prediction of nonabdominal, abdominal subcutaneous, and visceral fat. Am J Clin Nutr 75, 683-688. 